\title{
CRIAÇÃO DE UM WEBSIG DO MUNICÍPIO DE MOSSORÓ, RN, UTILIZANDO A TECNOLOGIA ALOVMAP
}

\author{
Jarbas Nunes Vidal Filho \\ Graduando do Curso de Ciências da Computação - Universidade Federal Rural do \\ Semiárido (UFERSA). E-mail: jarbasfito@ hotmail.com. \\ Angélica Félix de Castro \\ Professora Adjunta do Curso de Ciências da Computação - Universidade Federal Rural do \\ Semiárido (UFERSA). E-mail: angelica@ufersa.edu.br. \\ Francisco Paulo de Freitas Neto \\ Graduando do Curso de Ciências da Computação - Universidade Federal Rural do \\ Semiárido (UFERSA). E-mail: xikao_kfofo@hotmail.com. \\ Roberto Alves da Costa Júnior \\ Graduando do Curso de Ciências da Computação - Universidade Federal Rural do \\ Semiárido (UFERSA). E-mail: roberto.alves.jr@hotmail.com. \\ Denis Freire Lopes Nunes \\ Graduando do Curso de Ciências da Computação - Universidade Federal Rural do \\ Semiárido (UFERSA). E-mail: denisfln@ gmail.com.
}

\section{RESUMO}

Este trabalho aborda a divulgação e a publicação de mapas digitais interativos através do software ALOV Map. O ALOV Map é um servidor de mapas gratuito que pode ser usado de duas formas: com uma arquitetura cliente ou uma arquitetura cliente/servidor. Ambas as arquiteturas permitem publicar mapas na Web e a arquitetura cliente permite que sejam produzidas aplicações stand alone. O objetivo principal desse trabalho consiste em desenvolver um WebSIG com dados digitais do município de Mossoró, RN, de forma que seja possível a qualquer pessoa acessar mapas da cidade e obter informações mais detalhadas sobre ela.

PALAVRAS-CHAVE: AlovMap, Servidor de Mapas, Mapas interativos.

\section{CREATION OF A WEBGIS OF THE MOSSORÓ CITY, USING THE ALOVMAP TECHNOLOGY}

\begin{abstract}
This work presents the divulgation and publishing of interactive digital maps through the ALOV Map software. ALOV Map is a free map server that can be used in two ways: a client architecture or a client/server architecture. Both architectures allow to publish maps on the Web and the client architecture allows the production stand alone applications. The main objective of this work is to develop a WebGIS with the Mossoró city data, where it is possible for anyone to access city maps and more detailed information about this city
\end{abstract}

KEY-WORDS: AlovMap, Maps Server, Interactives Maps. 


\section{CRIAÇÃO DE UM WEBSIG DO MUNICÍPIO DE MOSSORÓ, RN, UTILIZANDO A TECNOLOGIA ALOVMAP}

\section{INTRODUÇÃO}

O termo Sistemas de Informação Geográfica (SIG) é aplicado para sistemas que realizam o tratamento computacional de dados geográficos. A principal diferença de um SIG para um sistema de informação convencional é sua capacidade de armazenar tanto os atributos descritivos como as geometrias dos diferentes tipos de dados geográficos. Assim, para cada lote num cadastro urbano, um SIG guarda, além de informação descritiva como proprietário e valor do IPTU, a informação geométrica com as coordenadas dos limites do lote (Casanova et al. 2005). A Figura 1 exibe a arquitetura de um SIG.

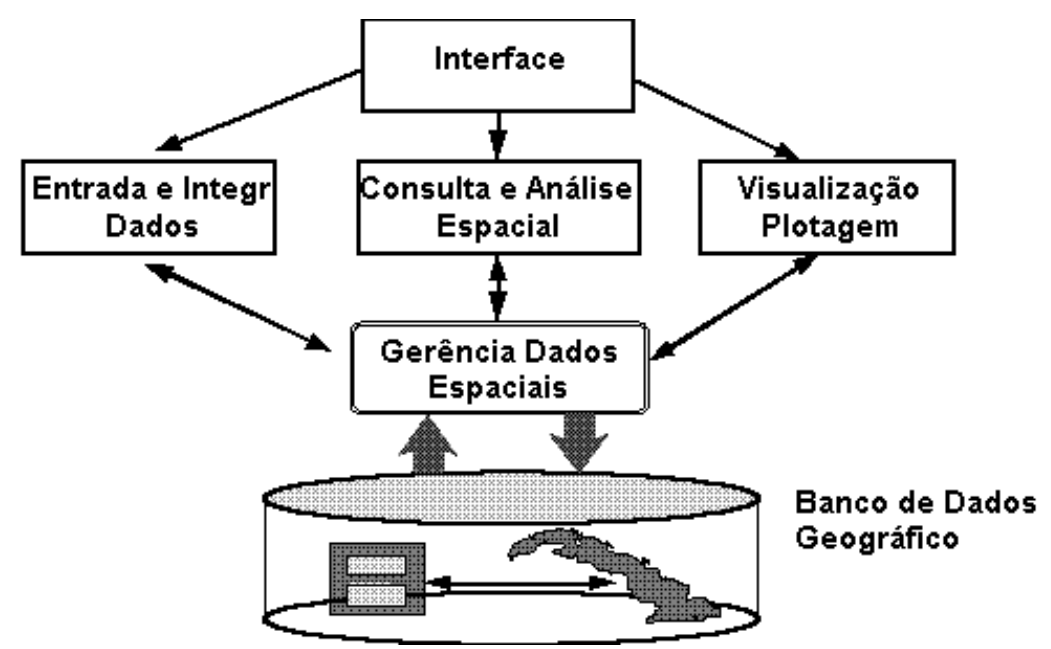

Figure 1 - Arquitetura de um SIG. (Fonte: INPE 2010).

Os novos mercados da Internet têm sido apontados como uma das principais razões para a atual expansão dos SIGs e da sua utilização nas mais diversas vertentes, transformando os SIGs num meio de comunicação ao fornecerem informação e conhecimento ao público (Yang et al. 2004). O papel dos WebSIG é remover estes significativos impedimentos do uso efetivo dos SIGs. O objetivo é que os usuários possam ser mais produtivos na aplicação das tecnologias SIG, eliminando o tempo dispendido no manuseamento de software e recolha de dados. O WebSIG permite ao usuário requisitar um serviço, que inclui os dados e as ferramentas de análise, de qualquer lugar usando a internet.

O WebSIG dos Sistemas de Informações Geográficas podem ser definidos como um conjunto de serviços de informação geográfica para a Internet, baseado numa rede que, utiliza diferentes formas de acesso à Internet para disponibilizar informação geográfica, ferramentas analíticas e diferentes serviços SIG (Peng et al. 2003). A disponibilidade e divulgação dos temas de forma dinâmica pela Internet, através do WebSIG, permite integrar, disseminar e comunicar informações geográficas visualmente na Internet.

Com o WebSIG é possível gerar páginas dinâmicas que permitem ao usuário consultas mais objetivas exclusivamente dedicadas aos temas do seu interesse (Mangabeira et al. 2002). O WebSIG combina duas poderosas tecnologias: os SIGs e a Internet, fornecendo conectividade em nível global. O resultado desta sinergia resulta numa maior facilidade em 
encontrar dados, partilhar ferramentas analíticas e o fato de ambos poderem chegar a um número maior de usuários (Tang et al. 2003).

Para que um SIG possa ser disponibilizado na internet, é necessário um servidor de mapas, que processa os pedidos dos clientes e gera resultados. O servidor de mapas executa as pesquisas espaciais, conduz a análise espacial, gerando e transmitindo os mapas para o cliente baseados nos pedidos do usuário. Este componente fornece funções tradicionais específicas dos SIG que incluem: filtros de pesquisa, serviços de geocodificação, análise espacial, criação de mapas, entre muitas outras.

Existem alguns servidores de mapas de destaque no mundo das informações geográficas, cada um com as suas características, vantagens e desvantagens. Os mais conhecidos são: MapGuide Open Source, MapBender, Deegree, AlovMap, Geoserver, Mapserver, entre outros.

O principal objetivo desse trabalho consiste em desenvolver um WebSIG para o município de Mossoró, RN, Brasil. Mossoró é a segunda cidade mais importante do Estado do Rio Grande do Norte, considerada uma das principais cidades do interior nordestino, onde atualmente vive um intenso crescimento econômico e de infraestrutura: o município é o maior produtor em terra, de petróleo no país, como também de sal marinho. A fruticultura irrigada, voltada em grande parte para a exportação, também possui relevância na economia estadual, tendo um dos maiores PIB per capita do estado. É tida como uma cidade de médio porte brasileira, sendo atraente para investimentos.

\section{TRABALHOS RELACIONADOS}

Alguns trabalhos foram desenvolvidos usando a tecnologia WebSIG. Atualmente, os servidores de mapas mais utilizados são o GeoServer, MapServer e o AlovMap.

Ramos (2009) utilizou a tecnologia GeoServer para disponibilizar para a comunidade portuguesa (e por que não, mundial?) a região hidrográfica do Alentejo, sul de Portugal, principalmente para avaliar as áreas de captação de água. Martins e Rocha (2009) desenvolveram uma plataforma WebSIG usando GeoServer para identificar áreas focos de uma determinada doença contagiosa.

A tecnologia MapServer encontra-se em alguns trabalhos interessantes: em Portugal, foi implementado um projeto Algarve Digital, onde toda a região do Algarve foi mapeada e disponibilizada em ambiente Web (Lehodey et al. 2008). No Brasil, o destaque é o geoportal desenvolvido pela Prefeitura de Ponta Grossa, Paraná, que permite ao usuário consultar informações georeferenciadas e tabulares sobre o município de modo interativo, através da manipulação de diferentes níveis de informação (camadas), de acordo com seu interesse e necessidade (GeoPortal 2010).

Melo (2006) disponibilizou alguns mapas interativos utilizando a tecnologia do AlovMap. Esse servidor de mapas é uma aplicação construída usando-se a linguagem Java que permite visualizar mapas tanto em formato vetorial do tipo shapefile (*.shp) como também raster através da internet, com qualidade bastante satisfatória. É um aplicativo gratuito e possibilita a publicação de dados e/ou mapas na Internet. Permite uma boa navegação e interação com os mapas, legendas, links na Internet, entre outras vantagens. Pode, ainda, ser utilizado de duas formas: com arquitetura cliente ou arquitetura cliente/servidor. 
A arquitetura cliente, mais simples, é executada no computador local, como um applet. Desta forma, o applet e todos os mapas são carregados do servidor para execução pela Máquina Virtual Java (Java Virtual Machine - JVM) residente no navegador. A arquitetura cliente/servidor é mais flexível e permite o envio de dados do servidor para o cliente de forma incremental. Os atributos do mapa são armazenados em um banco de dados padrão SQL. Sendo assim, se faz necessário o uso de um SGBD. O ALOV Map pode ser usado com os seguintes bancos de dados: Interbase, MySQL, Access e Hypersonic.

\section{METODOLOGIA}

Primeiramente foi realizado um mapeamento na cidade de Mossoró: foram mapeados os principais pontos da cidade para fase de teste e esse mapeamento continua em andamento, inclusive na porção conhecida como "Novo Mossoró", onde se localizam o shopping center, universidades particulares, grandes supermercados, entre outras. Foram utilizados os SIGs ArcView e gvSIG para mapear os principais pontos da cidades. O resultado desse mapeamento pode ser visto na Figura 2.

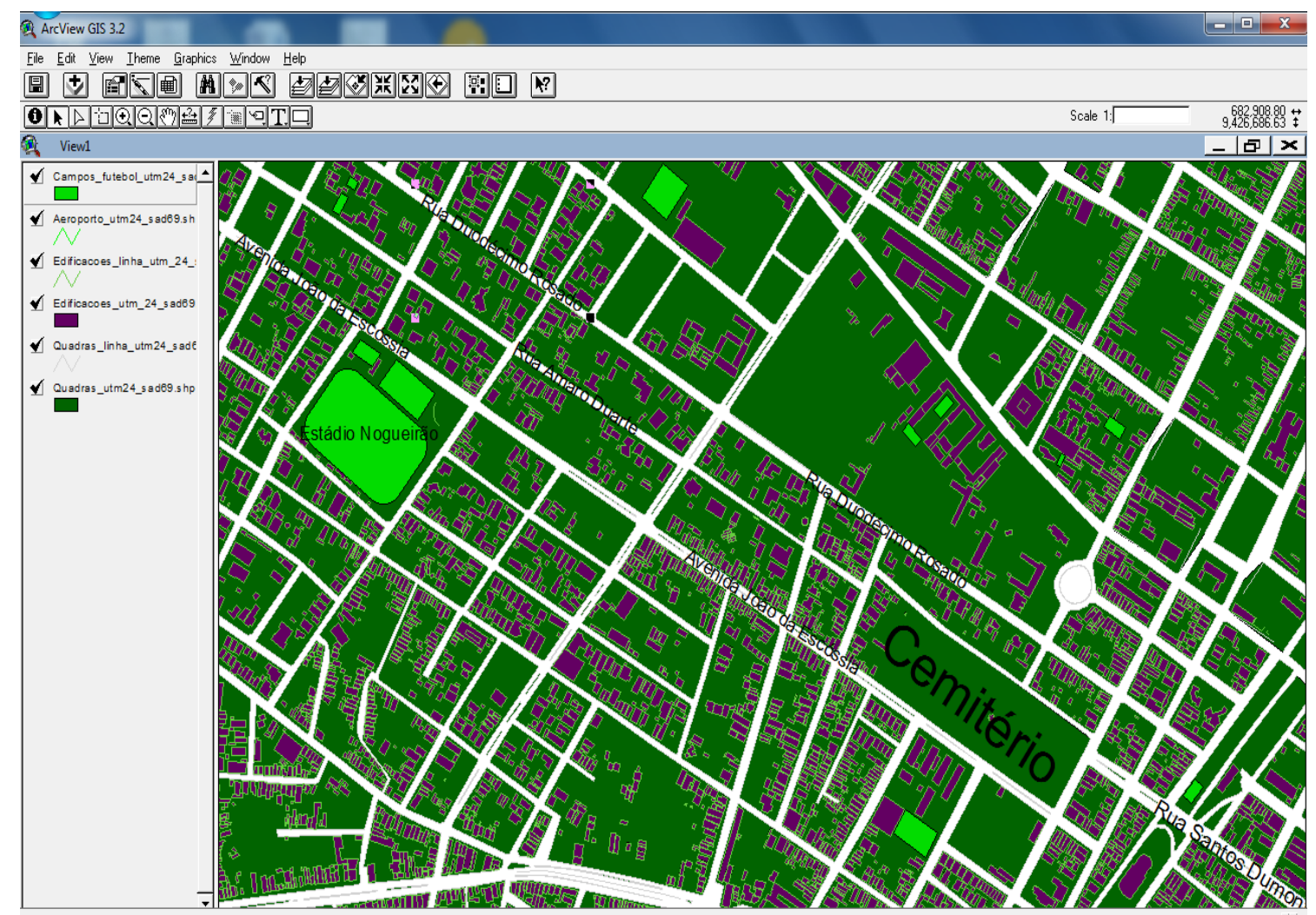

Figura 2 - Mapeamento da cidade de Mossoró no ArcView.

Realizado o mapeamento da cidade, foram comparados diversos servidores de mapas e decidiu-se por usar o servidor AlovMap, que dá suporte na publicação de mapas na internet. Como citado anteriormente, o AlovMap aborda duas arquiteturas: nesse trabalho, optou-se por utilizar uma arquitetura cliente, pois, dos vários trabalhos pesquisados, nenhum implementou nesse nível. Logo, trata-se de algo novo e inédito.

Essa arquitetura utiliza-se do armazenamento dos mapas em uma área pública do servidor e é composto por um arquivo de configuração XML (eXtensible Markup Language). Nesse arquivo é possível editar todas as configurações do sistema SIG, carregar os arquivos 
referentes aos mapas, definir o domínio da aplicação SIG, definir as cores, definir as legendas do sistema, entre outras operações que são essenciais para o desenvolvimento de um WebSIG por completo.

Existem dois arquivos: um applet e um arquivo HTML (HyperText Markup Language), o arquivo applet é o arquivo do servidor de mapas, ele é carregado dentro de um arquivo HTML, então utiliza-se a linguagem de marcação de hipertexto para carregar o arquivo applet e o arquivo de configuração XML.

A aplicação vai ser rodada em um arquivo HTML, pois é nela onde está sendo carregado o applet e o arquivo de configuração XML. Para finalizar essa aplicação, foi desenvolvida uma página Web, onde essa página mostra alguns conceitos sobre WebSIG, fala um pouco sobre a ferramenta e os plugins necessários para execução, já que a aplicação utiliza a JVM, além de ter um direcionamento para a aplicação SIG. Esse resultado pode ser visualizado na Figura 3.

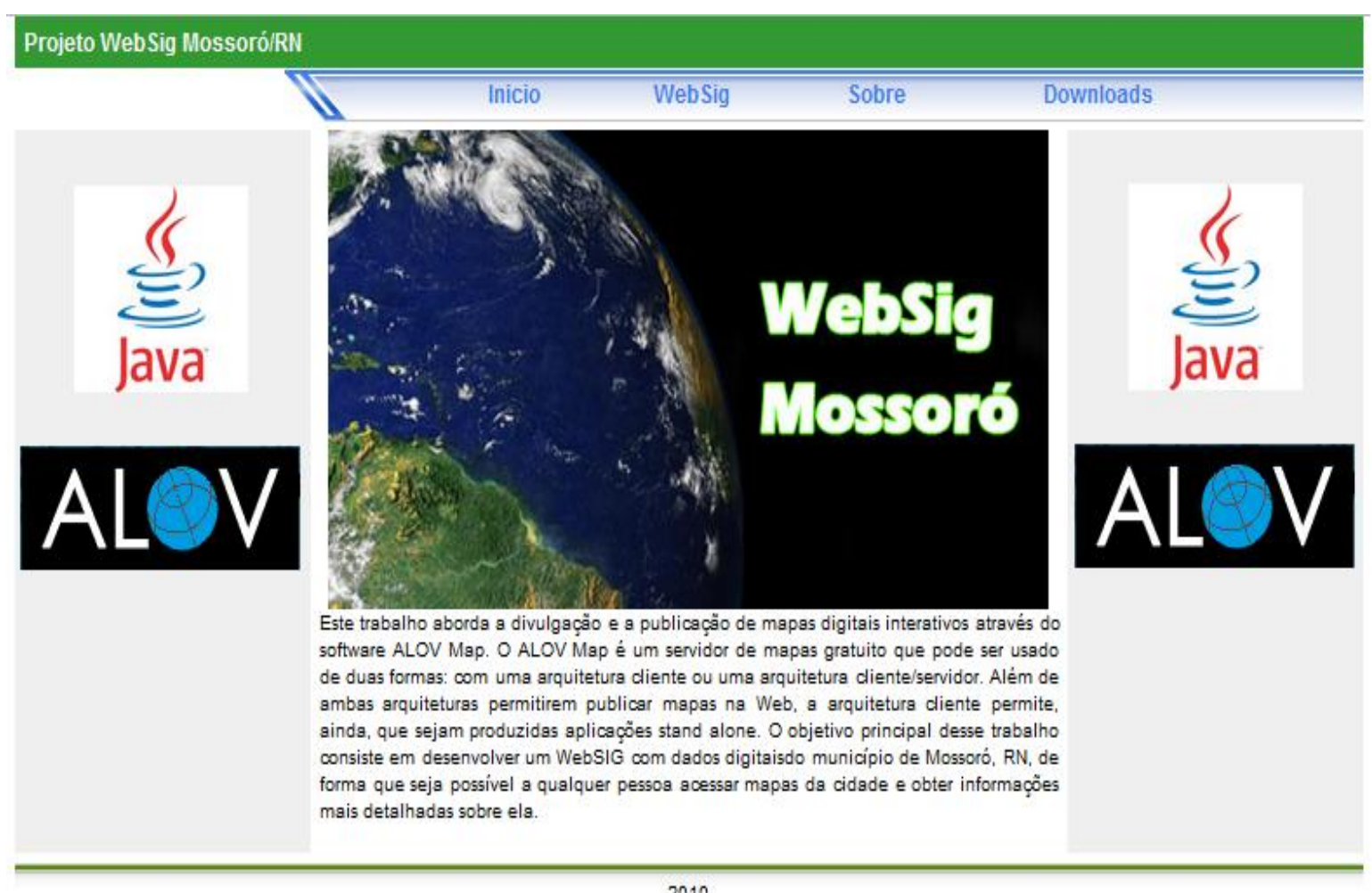

Figura 3 - Página Web do WebSIG.

\section{RESULTADOS OBTIDOS}

O resultado obtido nesse trabalho foi um WebSIG do munícipio de Mossoró, no Rio Grande do Norte. Esse sistema permite a visualização dos prédios administrativos, teatros, pavimentação, Universidades e outros. Além disso, é possível encontrar informações a respeito de prédios, como o tamanho da área, ano de construção, local que se encontra, bairro, entre outras.

Essa aplicação foi desenvolvida em forma de camadas, representadas em forma de legenda, onde o usuário pode selecionar qual área quer visualizar, pode realizar pesquisa para saber a localização da prefeitura da cidade, por exemplo, localizar as principais avenidas e ruas 
de Mossoró, mover o mapa para direita, esquerda, para cima, para baixo e aplicar Zoom em todo o mapa, a Figura 4 ilustra o sistema disponível na Web atualmente.

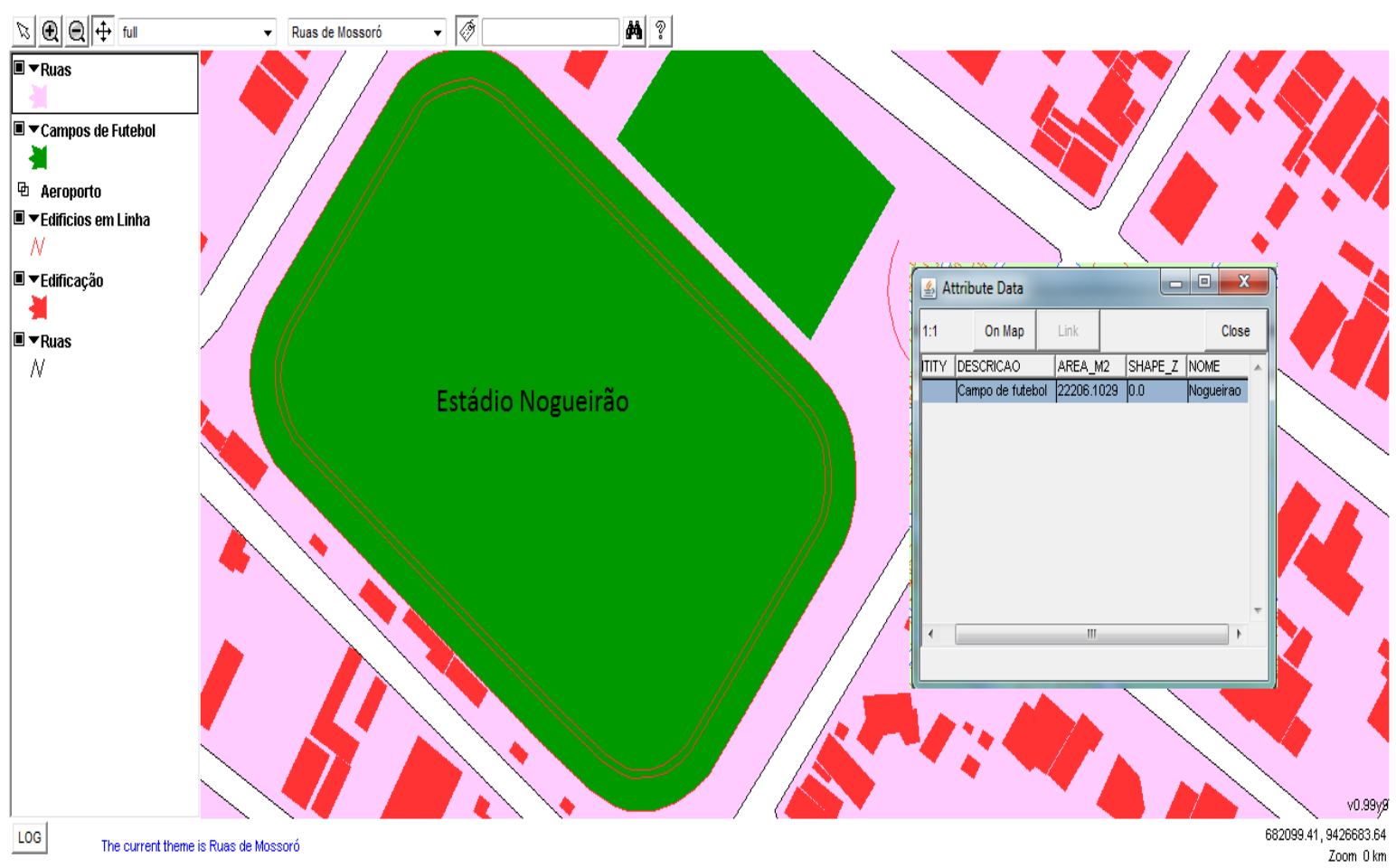

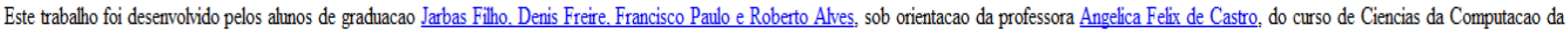
Universidade Federal do Semiarido.

\section{Figura 4 - Visualização do mapa de Mossoró e informações sobre o Estádio} Nogueirão (consulta feita pelo usuário) no WebSIG.

\section{CONCLUSÕES}

Os resultados obtidos nesse trabalho foram satisfatórios: foi obtido como produto final, um Sistema de Informações Geográficas que irá auxiliar as pessoas que moram na cidade de Mossoró, aos turistas, empresas e as demais pessoas que quiserem se localizar na cidade, pois a cidade ainda tem problemas no seu mapeamento urbano. O AlovMap foi uma tecnologia eficiente no desenvolvimento desse WebSIG.

Em trabalhos futuros, pretende-se desenvolver novas funcionalidades para o sistema. Dentre essas novas funcionalidades pretende-se implementar um serviço de rotas aos usuários e serviços que possam auxiliar os departamentos de segurança a minimizar a violência na cidade: esse serviço irá informar quais os pontos e horários em que há maior índice de violência, além de ajudar nos eventos festivos, como o São João que ocorre todos os anos na cidade de Mossoró, com grandes índices de criminalidade, assaltos, homicídios e acidentes.

\section{REFERÊNCIAS}

1. CASANOVA, M., CÂMARA, G., DAVIS, C., VINHAS, L. e QUEIROZ, G.R. Bancos de Dados Geográficos, Curitiba, MundoGEO. 2005. 
2. GeoPortal. GeoPortal Ponta Grossa - Divulgando nosso território, http://geo.pg.pr.gov.br/portal/, Ago. 2010.

3. LEHODEY, F., SENA, R., e FERREIRA, K. Mapas Interactivos do Algarve Implementação de uma plataforma WebSIG regional no âmbito do projecto Algarve Digital, www.algarvedigital.pt/algarve/index.php, 2008.

4. MANGABEIRA, J.A.D.C., CARVALHO, C.A.D. a OSHIRO, O.T. Disponibilização de Informações do Uso das Terras em Holambra com WebGIS, http://www.holambra.cnpm.embrapa.br/download/webgis_holambra.pdf, 2002.

5. MARTINS, H. e ROCHA, J. Portal SIGLA - uma plataforma WebSIG Open Source para vigilância entomológica da Língua Azul, In: 2nd Meeting of Free Software for GIS, 2009.

6. MELO, D.R. Divulgação e publicação de mapas digitais interativos com AlovMap, 71 p, 2006.

7. PENG, Z.-R. and TSOU, M.-H. Internet GIS - Distributed Geographic Information Services for the Internet and Wireless Networks (New Jersey: Wiley - John Wiley \& Sons, Inc.), 2003.

8. RAMOS, A. M. M. S. D. Disponibilização de informação geográfica na administração da região hidrográfica do Alentejo usando WEBSERVICES: WFS sobre GeoServer, http://run.unl.pt/handle/10362/2333, Ago, 2009.

9. TANG, W. e SELWOOD, J. Connecting our World - GIS Web Services. Redlands: ESRI Press, 2003.

10. YANG, C.P., WONG, D.W., YANG, R., KAFATOS, M. and LI, Q. Performanceimproving techniques in web-based GIS. International Journal of Geographical Information Science, 19:3, pp. 319-342, 2004. 